\title{
Representations of a $\mathbb{Z} / 3 \mathbb{Z}$-Quantum Group
}

\author{
Dedicated to Professor Noriaki Kawanaka on his 60th birthday
}

By

Hiroyuki YAMANE*

\begin{abstract}
In this paper, we give a classification of the finite dimensional irreducible representations of a nonstandard quantum group $U$, which may be viewed as a $\mathbb{Z} / 3 \mathbb{Z}$-graded version of a quantum superalgebra.
\end{abstract}

\section{Introduction}

Since the quantum groups, also called the quantum algebras, were introduced by Drinfeld [1] and Jimbo [5] in the mid 1980s, they have played important roles in study of statistical mechanics, knot theory, conformal field theory, and so on (cf. [19]). Moreover, the super-versions of quantum groups (see [7], [18]), called the quantum superalgebras, have also played important roles. For instance, they have been used to give new invariants of knots and links, which are distinct from well-known invariants associated with quantum algebras (see [10], [16]). So it would be interesting and important to look for and study new-versions of quantum groups.

In this paper, we introduce a new quantum group $U=U_{q}$, which can be viewed as a $\mathbb{Z} / 3 \mathbb{Z}$-graded version of a quantum superalgebra (see Remark 3 ). As a Hopf algebra, $U$ is not isomorphic to any of quantum algebras and quantum superalgebras (see Remark 7). Our main result of this paper is Theorem 4.1, which gives a classification of the finite dimensional irreducible representations

Communicated by M. Kashiwara. Received October 5, 2005.

2000 Mathematics Subject Classification(s): Primary 17B37; Secondary 17B10.

* Department of Pure and Applied Mathematics, Graduate School of Information Science and Technology, Osaka University, Toyonaka 560-0043, Japan.

e-mail: yamane@ist.osaka-u.ac.jp 
of $U$. (The positive part $U^{+}$(see $\S 1$ ) of $U$ has already been known (see [2]). It might be true that there exist only a few 'nonstandard' quantum groups other than $U$ (see [3]). We notice that $U$ is closely related to an anyonic Hopf algebra (see [13]).)

Let us explain the content of the paper more precisely. To prove Theorem 4.1, we use a method similar to Kac's proof [8, Theorem 8] of the classification of the finite dimensional irreducible representations of the simple Lie superalgebras (see also Remark 1). Among quantum superalgebras, the one resembling our $U$ most is the quantum superalgebra $U_{q} \operatorname{osp}(3 \mid 2)$. In order to show how $U$ resembles $U_{q} \operatorname{osp}(3 \mid 2)$, we explain in Remark 10 that we can also classify the finite dimensional irreducible representations of $U_{q} \operatorname{osp}(3 \mid 2)$ in a way similar to that for Theorem 4.1 (the classification result for $U_{q} \operatorname{osp}(3 \mid 2)$ seems to have been well-known to experts).

In Remark 8, we introduce a finite dimensional version $\mathbf{u}=\mathbf{u}_{\zeta}$ of $U$, where $\zeta$ is a root of unity. We show that $\mathbf{u}$ is a quasi-triangular Hopf algebra by giving an explicit formula of the universal $R$-matrix of $\mathbf{u}$. In Remark 9 , we discuss Drinfeld's topological version of $U$.

The paper is organized as follows. In $\S 1$, we introduce $U$ and give a PBWbasis of the positive part $U^{+}$of $U$ (see Lemma 1.2). In $\S 2$, we introduce irreducible highest weight $U$-modules $\mathcal{L}\left(a, b_{1}, b_{2}\right)\left(=\mathcal{L}(\lambda)\right.$, where $\lambda=\left(a, b_{1}, b_{2}\right)$ (see $(2.10)))$. In $\S 3$, we study small $U$-modules. In $\S 4$, we state and prove our main result Theorem 4.1. In $\S 5$, we give some additional remarks.

We explain the strategy of the proof of our main theorem.

Remark 1. Strategy of the proof of our main theorem Theorem 4.1. We use the fact that $U$ contains two subalgebras $V_{1}$ and $V_{2}$ isomorphic to $\mathrm{sl}_{2}$ quantum algebras (precisely, $V_{1} \simeq U_{\omega^{2} q^{-1} \mathrm{sl}_{2}}$ and $V_{2} \simeq U_{q} \mathrm{sl}_{2}$ ) (see Remark 5). Roughly speaking, a part obtained from $U$ by excluding $V_{1}$ and $V_{2}$ is finite dimensional (see Lemma 1.2 and Lemma $1.1(1)-(2)$ ). We find the finite dimensional modules from the irreducible highest weight modules $\mathcal{L}(\lambda)=U v_{\lambda}$ (see (2.10) for $\mathcal{L}(\lambda)$ and the highest weight vector $v_{\lambda}$ ). To do so, we find the condition that $\operatorname{dim} V_{i} v_{\lambda}<\infty$ for $i=1,2$ (see Lemmas 2.2 and 4.1).

\section{$\S 1$. Definition of $U$}

Let $\mathbb{C}$ be the field of the complex numbers and $\mathbb{Z}_{+}=\{n \in \mathbb{Z} \mid n \geq 0\}$. Then $\mathbb{Z}_{+}=\mathbb{N} \cup\{0\}$. Let $\mathbb{K}=\mathbb{C}\left(q^{\frac{1}{2}}\right)$ be the field of the rational functions in an indeterminate $q^{\frac{1}{2}}$. Let $\mathbb{K}^{\times}=\mathbb{K} \backslash\{0\}$. Let $\omega=\frac{-1+\sqrt{-3}}{2}\left(\in \mathbb{K}^{\times}\right)$, i.e., 
$\omega^{2}+\omega+1=0$ and $\omega$ is a primitive third root of unity. Let

$$
A=\left(\begin{array}{ll}
a_{11} & a_{12} \\
a_{21} & a_{22}
\end{array}\right)=\left(\begin{array}{cc}
0 & -1 \\
-1 & 2
\end{array}\right) .
$$

Let $p(1)=1$ and $p(2)=0$.

Let $U=U_{q}$ be a $\mathbb{K}$-algebra defined with generators

$$
\sigma, K_{1}^{ \pm 1}, K_{2}^{ \pm 1}, E_{1}, E_{2}, F_{1}, F_{2}
$$

and relations

$$
\begin{gathered}
\sigma^{3}=1, K_{i} K_{i}^{-1}=K_{i}^{-1} K_{i}=1, K_{i} K_{j}=K_{j} K_{i} \\
\sigma K_{i} \sigma^{-1}=K_{i}, \sigma E_{i} \sigma^{-1}=\omega^{p(i)} E_{i}, \sigma F_{i} \sigma^{-1}=\omega^{-p(i)} F_{i} \quad(i \in\{1,2\}), \\
K_{i} E_{j} K_{i}^{-1}=q^{a_{i j}} E_{j}, K_{i} F_{j} K_{i}^{-1}=q^{-a_{i j}} F_{j} \quad(i, j \in\{1,2\}), \\
E_{i} F_{j}-F_{j} E_{i}=\delta_{i j}\left(-L_{i}+L_{i}^{-1}\right) \quad(i, j \in\{1,2\}) \\
E_{1}^{3}=0, E_{2}^{2} E_{1}-\left(q+q^{-1}\right) E_{2} E_{1} E_{2}+E_{1} E_{2}^{2}=0 \\
F_{1}^{3}=0, F_{2}^{2} F_{1}-\left(q+q^{-1}\right) F_{2} F_{1} F_{2}+F_{1} F_{2}^{2}=0
\end{gathered}
$$

where we let $L_{i}=K_{i} \sigma^{p(i)}$.

Remark 2. As for (1.5), we do not let RHS be $\delta_{i j} \frac{L_{i}-L_{i}^{-1}}{q-q^{-1}}$. The reason that we let it be $\delta_{i j}\left(-L_{i}+L_{i}^{-1}\right)$ is that it is natural from the viewpoint of the quantum double construction (see [1], [15], [4, Proposition 6.12 (2)] and [18, Proposition 2.5.1 (2.5.5) and Theorem 2.9.4 (ii)]).

Define an automorphism $\Omega: U \rightarrow U$ by $\Omega\left(K_{i}\right)=K_{i}^{-1}, \Omega(\sigma)=\sigma^{-1}$, $\Omega\left(E_{i}\right)=F_{i}$ and $\Omega\left(F_{i}\right)=E_{i}$. Then $\Omega^{2}=\mathrm{id}_{U}$.

We regard $U$ as a Hopf algebra $(U, \Delta, S, \varepsilon)$ defined by

$$
\left\{\begin{array}{l}
\Delta(\sigma)=\sigma \otimes \sigma, \Delta\left(K_{i}^{ \pm 1}\right)=K_{i}^{ \pm 1} \otimes K_{i}^{ \pm 1}, \\
\Delta\left(E_{i}\right)=E_{i} \otimes 1+L_{i} \otimes E_{i}, \Delta\left(F_{i}\right)=F_{i} \otimes L_{i}^{-1}+1 \otimes F_{i}, \\
S(\sigma)=\sigma^{-1}, S\left(K_{i}\right)=K_{i}^{-1}, S\left(E_{i}\right)=-L_{i}^{-1} E_{i}, S\left(F_{i}\right)=-F_{i} L_{i}, \\
\varepsilon(\sigma)=1, \varepsilon\left(K_{i}\right)=1, \varepsilon\left(E_{i}\right)=0, \varepsilon\left(F_{i}\right)=0 .
\end{array}\right.
$$


Remark 3. Let $U^{(i)}=\left\{X \in U \mid \sigma X \sigma^{-1}=\omega^{i} X\right\}$ for $i \in \mathbb{Z}$. Then $U$ is a $\mathbb{Z} / 3 \mathbb{Z}$-graded Hopf algebra with respect to $U^{(i)}$ 's, namely, $U=U^{(0)} \oplus$ $U^{(1)} \oplus U^{(2)}, U^{(i+3)}=U^{(i)}, U^{(i)} U^{(j)} \subset U^{(i+j)}, \Delta\left(U^{(i)}\right) \subset \oplus_{x+y=i} U^{(x)} \otimes U^{(y)}$, $S\left(U^{(i)}\right)=U^{(i)}, \varepsilon\left(U^{(0)}\right)=\mathbb{K}$ and $\varepsilon\left(U^{(1)} \oplus U^{(2)}\right)=\{0\}$.

Let $U^{0}$ be the subalgebra of $U$ generated by $\sigma, K_{1}^{ \pm 1}$ and $K_{2}^{ \pm 1}$. Let $U^{+}$ (resp. $U^{-}$) be the subalgebra (with 1 ) of $U$ generated by $E_{1}$ and $E_{2}$ (resp. $F_{1}$ and $F_{2}$ ). For $m, n \in \mathbb{Z}_{+}$, we define the $\mathbb{K}$-linear subspaces $U_{m, n}^{+}$of $U^{+}$in the following way; we first set $U_{0,0}^{+}:=\mathbb{K}, U_{m, 0}^{+}:=\mathbb{K} E_{1}^{m}$ and $U_{0, n}^{+}:=\mathbb{K} E_{2}^{n}$ and then, for $m \geq 1$ and $n \geq 1$, we set $U_{m, n}^{+}:=E_{1} U_{m-1, n}^{+}+E_{2} U_{m, n-1}^{+}$inductively.

For a subset $\mathcal{S}$ of $U$, let $\operatorname{Span}_{\mathbb{K}}(\mathcal{S})=\sum_{s \in \mathcal{S}} \mathbb{K} s$. Then $\operatorname{Span}_{\mathbb{K}}\left(U^{+} U^{0}\right)$ is the subalgebra of $U$ generated by $U^{0}$ and $U^{+}$. We also notice that $\operatorname{Span}_{\mathbb{K}}\left(U^{+} U^{0}\right)=$ $\operatorname{Span}_{\mathbb{K}}\left(U^{0} U^{+}\right)$and $\operatorname{Span}_{\mathbb{K}}\left(U^{-} U^{0}\right)=\Omega\left(\operatorname{Span}_{\mathbb{K}}\left(U^{+} U^{0}\right)\right)$.

By a well-known argument (see [1], [4, Theorem 4.21 and Proposition 6.12] (see the second paragraph of Remark 8), [14, II. Proposition 2], [15, II.1. Lemma 1], [17, Proposition 2.3] and [18]), we have the following.

Lemma 1.1. (1) As a $\mathbb{K}$-linear space,

$$
U \simeq U^{+} \otimes U^{0} \otimes U^{-} \quad(X Z Y \leftarrow X \otimes Z \otimes Y)
$$

and

$$
U \simeq U^{-} \otimes U^{0} \otimes U^{+} \quad(Y Z X \leftarrow Y \otimes Z \otimes X) .
$$

(2) The set $\left\{K_{1}^{n_{1}} K_{2}^{n_{2}} \sigma^{n_{3}} \mid n_{1}, n_{2} \in \mathbb{Z}, n_{3} \in\{0,1,2\}\right\}$ is a $\mathbb{K}$-basis of $U^{0}$.

(3) As a $\mathbb{K}$-algebra (with 1$), U^{+}$can also be defined with the generators $E_{1}$ and $E_{2}$ and the relations (1.6). In particular,

$$
U^{+}=\bigoplus_{m, n=0}^{\infty} U_{m, n}^{+}
$$

and $\operatorname{dim} U_{0,0}^{+}=\operatorname{dim} U_{1,0}^{+}=\operatorname{dim} U_{0,1}^{+}=1$.

(4) There exists a unique symmetric bilinear form

$$
(,): \operatorname{Span}_{\mathbb{K}}\left(U^{+} U^{0}\right) \times \operatorname{Span}_{\mathbb{K}}\left(U^{+} U^{0}\right) \rightarrow \mathbb{K}
$$

which satisfies the conditions (i)-(v) below.

(i) For any $X, Y, Z \in \operatorname{Span}_{\mathbb{K}}\left(U^{+} U^{0}\right)$, the equation

$$
(X Y, Z)=(X \otimes Y, \Delta(Z))
$$

holds. 
(ii) For any $X_{1}, X_{2} \in U^{+}$and any $Z_{1}, Z_{2} \in U^{0}$, the equation

$$
\left(X_{1} Z_{1}, X_{1} Z_{2}\right)=\left(X_{1}, X_{2}\right)\left(Z_{1}, Z_{2}\right)
$$

holds.

(iii) For any $c(1), c(2), d(1), d(2) \in \mathbb{Z}$ and any $c(3), d(3) \in\{0,1,2\}$, the equation

$$
\left(K_{1}^{c(1)} K_{2}^{c(2)} \sigma^{c(3)}, K_{1}^{d(1)} K_{2}^{d(2)} \sigma^{d(3)}\right)=q^{\sum_{i=1}^{2} \sum_{j=1}^{2} c(i) a_{i j} d(j)} \omega^{c(3) d(3)}
$$

holds.

(iv) For any $i, j \in\{1,2\}$, the equation

$$
\left(E_{i}, E_{j}\right)=\delta_{i j}
$$

holds.

(v) For any $m, n, r, l \in \mathbb{Z}_{+}$with $m \neq r$ or $n \neq l$, the equation

$$
\left(U_{m, n}^{+}, U_{r, l}^{+}\right)=\{0\}
$$

holds.

Let

$$
\begin{gathered}
E_{12}=E_{1} E_{2}-q E_{2} E_{1}, \quad E_{112}=E_{1} E_{12}-\omega^{2} q E_{12} E_{1}, \\
F_{12}=\frac{1}{q^{2}-1} \Omega\left(E_{12}\right)=\frac{1}{q^{2}-1}\left(F_{1} F_{2}-q F_{2} F_{1}\right)
\end{gathered}
$$

and

(1.11) $\quad F_{112}=-\frac{1}{\left(q^{2}-1\right)\left(q^{2}-\omega\right)} \Omega\left(E_{112}\right)=-\frac{1}{q^{2}-\omega}\left(F_{1} F_{12}-\omega^{2} q F_{12} F_{1}\right)$.

Then we have the following (see also Remark 4 below and notice $\Omega\left(U^{+}\right)=U^{-}$).

$$
E_{12} E_{2}=q^{-1} E_{2} E_{12}, \quad F_{12} F_{2}=q^{-1} F_{2} F_{12}
$$

$E_{112} E_{2}-E_{2} E_{112}=-q^{-1} \omega^{2}\left(q^{2}-\omega\right) E_{12}^{2}, \quad F_{112} F_{2}-F_{2} F_{112}=q^{-1} \omega^{2}\left(q^{2}-1\right) F_{12}^{2}$

$$
\begin{gathered}
E_{112} E_{12}=\omega q E_{12} E_{112}, \quad F_{112} F_{12}=\omega q F_{12} F_{112} \\
E_{12}^{3}=0, \quad F_{12}^{3}=0
\end{gathered}
$$


Remark 4. Proof of (1.15)-(1.16). We let $[X, Y]_{t}=X Y-t Y X$.

We first prove the first equality of (1.15). We have

$$
\begin{aligned}
0 & =\left[E_{1}, E_{112} E_{2}-E_{2} E_{112}+q^{-1} \omega^{2}\left(q^{2}-\omega\right) E_{12}^{2}\right]_{\omega q^{2}} \quad(\text { by }(1.14)) \\
& =\omega q E_{112} E_{12}-E_{12} E_{112}+q^{-1} \omega^{2}\left(q^{2}-\omega\right)\left(E_{112} E_{12}+\omega^{2} q E_{12} E_{112}\right) \quad(\text { by }(1.13)) \\
& =\left(q+q^{-1}\right)\left(-E_{112} E_{12}+\omega q E_{12} E_{112}\right),
\end{aligned}
$$

which implies the desired equality.

Next, we prove the first equality of (1.16). We have

$$
\begin{aligned}
0 & =\left[\left[\left[E_{1}^{3}, E_{2}\right]_{q^{3}}, E_{2}\right]_{q}, E_{2}\right]_{q^{-1}} \quad(\text { by the first equality of }(1.6)) \\
& =\left[\left[E_{1}^{2} E_{12}+q E_{1} E_{12} E_{1}+q^{2} E_{12} E_{1}^{2}, E_{2}\right]_{q}, E_{2}\right]_{q^{-1}} \\
& =\left(q+q^{-1}\right)\left(q^{2}+1+q^{-2}\right) E_{12}^{3} \quad(\text { by }(1.6))
\end{aligned}
$$

which implies the desired equality.

We can obtain the second equations of (1.15)-(1.16) from the first ones by applying $\Omega$.

We also have the following.

$$
\Delta\left(E_{12}\right)=E_{12} \otimes 1+\left(q^{-1}-q\right) E_{2} L_{1} \otimes E_{1}+L_{1} L_{2} \otimes E_{12}
$$

$$
\begin{aligned}
\Delta\left(E_{112}\right)= & E_{112} \otimes 1+\left(\omega q-\omega^{2} q^{-1}\right) E_{12} L_{1} \otimes E_{1} \\
& +\left(q^{-1}-q\right)\left(q^{-1}-\omega^{2} q\right) E_{2} L_{1}^{2} \otimes E_{1}^{2}+L_{1}^{2} L_{2} \otimes E_{112}
\end{aligned}
$$

$$
\text { Let } \Phi(n, t)=\prod_{i=1}^{n} \frac{1-t^{i}}{1-t} \text {. }
$$

Lemma 1.2. The set

$$
\left\{E_{1}^{n(1)} E_{112}^{n(112)} E_{12}^{n(12)} E_{2}^{n(2)} \mid n(112), n(2) \in \mathbb{Z}_{+}, n(1), n(12) \in\{0,1,2\}\right\}
$$

is a $\mathbb{K}$-basis of $U^{+}$.

Proof. By (1.12)-(1.16), we see that the set (1.21) spans $U^{+}$. We show that they form an orthogonal basis. By (1.12)-(1.18) and the same argument 
as in [15, II. Lemma 1], we have

$$
\begin{aligned}
( & \left.E_{1}^{m(1)} E_{112}^{m(112)} E_{12}^{m(12)} E_{2}^{m(2)}, E_{1}^{n(1)} E_{112}^{n(112)} E_{12}^{n(12)} E_{2}^{n(2)}\right) \\
= & \delta_{m(1), n(1)} \delta_{m(112), n(112)} \delta_{m(12), n(12)} \delta_{m(2), n(2)} \\
& . \Phi(m(1), \omega)\left(E_{1}, E_{1}\right)^{m(1)} \Phi\left(m(112), \omega q^{-2}\right)\left(E_{112}, E_{112}\right)^{m(112)} \\
& . \Phi(m(12), \omega)\left(E_{12}, E_{12}\right)^{m(12)} \Phi\left(m(2), q^{2}\right)\left(E_{2}, E_{2}\right)^{m(2)}
\end{aligned}
$$

Notice $\left(E_{1}, E_{1}\right)=\left(E_{2}, E_{2}\right)=1$ (see Lemma 1.1 (4) (iv)). By (1.19)-(1.20), $\left(E_{12}, E_{12}\right) \neq 0$ and $\left(E_{112}, E_{112}\right) \neq 0$. Then we have the desired result.

\section{§2. Verma Modules}

Notice $\Omega\left(U^{+}\right)=U^{-}$. We let $[X, Y]=X Y-Y X$. We have the following.

$$
\begin{gathered}
{\left[E_{1}, F_{12}\right]=-q^{-1} F_{2} L_{1}^{-1}, \quad\left[F_{1}, E_{12}\right]=-q^{-1}\left(q^{2}-1\right) E_{2} L_{1}} \\
{\left[E_{1}, F_{112}\right]=-\omega q^{-1} F_{12} L_{1}^{-1}, \quad\left[F_{1}, E_{112}\right]=\omega q^{-1}\left(q^{2}-\omega\right) E_{12} L_{1}} \\
{\left[E_{2}, F_{12}\right]=F_{1} L_{2}, \quad\left[F_{2}, E_{12}\right]=\left(q^{2}-1\right) E_{1} L_{2}^{-1}} \\
{\left[E_{2}, F_{112}\right]=\omega^{2} F_{1}^{2} L_{2}, \quad\left[F_{2}, E_{112}\right]=-\omega^{2}\left(q^{2}-1\right)\left(q^{2}-\omega\right) E_{1}^{2} L_{2}^{-1}} \\
{\left[E_{12}, F_{12}\right]=-L_{1} L_{2}+L_{1}^{-1} L_{2}^{-1}} \\
{\left[E_{12}, F_{112}\right]=F_{1} L_{1} L_{2}, \quad\left[F_{12}, E_{112}\right]=-\left(q^{2}-\omega\right) E_{1} L_{1}^{-1} L_{2}^{-1}} \\
{\left[E_{112}, F_{112}\right]=-L_{1}^{2} L_{2}+L_{1}^{-2} L_{2}^{-1}}
\end{gathered}
$$

Remark 5. By Lemma $1.1(1)-(2)$ and Lemma 1.2, using $\Omega$, we see that the elements

$$
E_{1}^{m(1)} E_{112}^{m(112)} E_{12}^{m(12)} E_{2}^{m(2)} K_{1}^{c(1)} K_{2}^{c(2)} \sigma^{c(3)} F_{1}^{n(1)} F_{112}^{n(112)} F_{12}^{n(12)} F_{2}^{n(2)}
$$

with $m(112), m(2), n(112), n(2) \in \mathbb{Z}_{+}, c(1), c(2) \in \mathbb{Z}$ and $0 \leq m(1), m(12), c(3)$, $n(1), n(12) \leq 2$ form a basis of $U$. Let $V_{1}$ (resp. $V_{2}$ ) be the subalgebra of $U$ generated by $\left(L_{1}^{2} L_{2}\right)^{ \pm 1}, E_{112}$ and $F_{112}$ (resp. $L_{2}^{ \pm 1}, E_{2}$ and $F_{2}$ ). By $(1.2)-(1.5)$ and (2.7), using the basis in (2.8), we see that as $\mathbb{K}$-algebras, $V_{1}$ and $V_{2}$ are isomorphic to $U_{\omega^{2} q^{-1}} \mathrm{sl}_{2}$ and $U_{q^{2}} \mathrm{sl}_{2}$ respectively. See also Remark 1 for $V_{1}$ and $V_{2}$. 
It follows from Lemma $1.1(1)-(3)$ that for $a \in\{0,1,2\}$ and $b_{1}, b_{2} \in \mathbb{K}^{\times}$, there exists a unique (left) $U$-module $\mathcal{M}\left(a, b_{1}, b_{2}\right)$ having the following properties.

(i) As a $U^{-}$-module, $\mathcal{M}\left(a, b_{1}, b_{2}\right)$ is free and rank one.

(ii) Let $v^{\mathcal{M}}$ be a basis element of the rank-one free $U^{-}$-module $\mathcal{M}\left(a, b_{1}, b_{2}\right)$, so $\mathcal{M}\left(a, b_{1}, b_{2}\right)=U^{-} v^{\mathcal{M}}$. Then $\sigma v^{\mathcal{M}}=\omega^{a} v^{\mathcal{M}}, K_{i} v^{\mathcal{M}}=b_{i} v^{\mathcal{M}}$ and $E_{i} v^{\mathcal{M}}=0$.

We call $\mathcal{M}\left(a, b_{1}, b_{2}\right)$ the Verma module with a highest weight $\left(a, b_{1}, b_{2}\right)$. Let $\mathcal{N}\left(a, b_{1}, b_{2}\right)$ be the maximal proper submodule of $\mathcal{M}\left(a, b_{1}, b_{2}\right)$. Let $\mathcal{L}\left(a, b_{1}, b_{2}\right)$ be the quotient module $\mathcal{M}\left(a, b_{1}, b_{2}\right) / \mathcal{N}\left(a, b_{1}, b_{2}\right)$. Then $\mathcal{L}\left(a, b_{1}, b_{2}\right)$ is irreducible and $\mathcal{L}\left(a, b_{1}, b_{2}\right) \neq\{0\}$. We let

$$
v\left(a, b_{1}, b_{2}\right)=v^{\mathcal{M}}+\mathcal{N}\left(a, b_{1}, b_{2}\right)
$$

$\left(\in \mathcal{L}\left(a, b_{1}, b_{2}\right)\right)$.

Abbreviations of $v\left(a, b_{1}, b_{2}\right)$ and $\mathcal{L}\left(a, b_{1}, b_{2}\right)$. For $\lambda=\left(a, b_{1}, b_{2}\right) \in\{0,1,2\} \times$ $\left(\mathbb{K}^{\times}\right)^{2}$, we let

$$
v_{\lambda}=v\left(a, b_{1}, b_{2}\right) \quad \text { and } \quad \mathcal{L}(\lambda)=\mathcal{L}\left(a, b_{1}, b_{2}\right) .
$$

We call $\mathcal{L}(\lambda)$ the irreducible highest weight $(U$-) module with a highest weight $\lambda$. We call $v_{\lambda}$ a highest weight vector of $\mathcal{L}(\lambda)$.

Let

$$
\mathcal{A}=\left\{\lambda \in\{0,1,2\} \times\left(\mathbb{K}^{\times}\right)^{2} \mid \operatorname{dim} \mathcal{L}(\lambda)<\infty\right\} .
$$

Similarly to [14, Proof of Proposition 3], using $V_{i}$ in Remark 5, we have:

Lemma 2.1. The $U$-modules $\mathcal{L}(\lambda), \lambda \in \mathcal{A}$, form a complete set of nonisomorphic finite dimensional irreducible $U$-modules (see (2.10) for $\mathcal{L}(\lambda)$ ).

For $r(1), r(2) \in \mathbb{Z}_{+}$, let $\mathcal{B}_{r(1), r(2)}$ be the subset of $\{0,1,2\} \times\left(\mathbb{K}^{\times}\right)^{2}$ formed by the elements

$$
\left(a, \epsilon_{1} \omega^{-a+r(1)} q^{-\frac{r(1)+r(2)}{2}}, \epsilon_{2} q^{r(2)}\right)
$$

with $a \in\{0,1,2\}, \epsilon_{1} \in\{ \pm 1, \pm \sqrt{-1}\}$ and $\epsilon_{2} \in\{ \pm 1\}$ (see also Remark 6 below).

Let $\mathcal{B}=\cup_{(r(1), r(2)) \in \mathbb{Z}_{+} \times \mathbb{Z}_{+}} \mathcal{B}_{r(1), r(2)}$.

Let $\lambda=\left(a, \epsilon_{1} \omega^{-a+r(1)} q^{-\frac{r(1)+r(2)}{2}}, \epsilon_{2} q^{r(2)}\right) \in \mathcal{B}_{r(1), r(2)}$ (see (2.11)) and let $v=v_{\lambda}$ (see (2.9)-(2.10)). For later use, we give the following formulas.

$$
L_{1} v=\epsilon_{1} \omega^{r(1)} q^{-\frac{r(1)+r(2)}{2}} v, L_{2} v=\epsilon_{2} q^{r(2)} v, L_{1}^{2} L_{2} v=\epsilon_{1}^{2} \epsilon_{2} \omega^{2 r(1)} q^{-r(1)} v
$$




$$
\begin{aligned}
& \left(L_{1}^{2} L_{2}\right)^{2} F_{1}^{n} v=\left(\omega q^{-2}\right)^{r(1)-n} F_{1}^{n} v \\
& \left(L_{1}^{2} L_{2}\right)^{2} F_{12}^{n} v=\left(\omega q^{-2}\right)^{r(1)-n} F_{12}^{n} v
\end{aligned}
$$

Remark 6. We have

$$
\begin{aligned}
& \mathcal{B}_{r(1), r(2)} \\
& =\left\{\left(a, b_{1}, b_{2}\right) \in\{0,1,2\} \times\left(\mathbb{K}^{\times}\right)^{2}\right. \\
& \left.\quad \mid b_{2}^{2}=\left(q^{2}\right)^{r(2)},\left(\left(\omega^{a} b_{1}\right)^{2} b_{2}\right)^{2}=\left(\left(\omega^{2} q^{-1}\right)^{2}\right)^{r(1)}\right\} .
\end{aligned}
$$

In order to see a significance of (2.15), recall Remark 5 and notice that for $v$ in (2.12), if $\lambda$ is also denoted as $\left(a, b_{1}, b_{2}\right)$, then $L_{2} v=b_{2} v$ and $L_{1}^{2} L_{2} v=$ $\left(\omega^{a} b_{1}\right)^{2} b_{2} v$.

\section{Lemma 2.2. $\mathcal{A} \subset \mathcal{B}$.}

Proof. (See also Remarks 1, 5 and 6.) Let $\lambda=\left(a, b_{1}, b_{2}\right) \in \mathcal{A}$ and $v=$ $v_{\lambda}(\in \mathcal{L}(\lambda))($ see $(2.9)-(2.10))$. Then, by (1.3)-(1.5), we have

$$
\begin{aligned}
E_{2} F_{2}^{m} v & =F_{2}^{m-1}\left(-\frac{1-q^{-2 m}}{1-q^{-2}} L_{2}+\frac{1-q^{2 m}}{1-q^{2}} L_{2}^{-1}\right) v \\
& =-\frac{1-q^{-2 m}}{1-q^{-2}} b_{2}^{-1}\left(b_{2}^{2}-q^{2(m-1)}\right) F_{2}^{m-1} v
\end{aligned}
$$

for $m \in \mathbb{N}$. Assume that $b_{2}^{2} \neq q^{2(m-1)}$ for all $m \in \mathbb{N}$. Then $F_{2}^{m} v \neq 0$ since $E_{2}^{m} F_{2}^{m} v \in \mathbb{K}^{\times} v$ by (2.16). Since $K_{2} F_{2}^{m} v=q^{-2 m} b_{2} F_{2}^{m} v, F_{2}^{m} v$ 's are linearly independent, which contradicts $\operatorname{dim} \mathcal{L}(\lambda)<\infty$. Then $b_{2}^{2}=q^{2 r(2)}$ for some $r(2) \in \mathbb{Z}_{+}$, so $b_{2}=\epsilon_{2} q^{r(2)}$ for some $\epsilon_{2} \in\{ \pm 1\}$.

By (2.7) and $L_{1}^{2} L_{2} v=\omega^{2 a} b_{1}^{2} b_{2} v$, similarly to (2.16), we have

$$
E_{112} F_{112}^{m} v=-\frac{1-\left(\omega q^{-2}\right)^{-m}}{1-\left(\omega q^{-2}\right)^{-1}}\left(\omega^{2 a} b_{1}^{2} b_{2}\right)^{-1}\left(\left(\omega^{2 a} b_{1}^{2} b_{2}\right)^{2}-\left(\omega q^{-2}\right)^{m-1}\right) F_{112}^{m-1} v .
$$

By the same argument as in the previous paragraph, $\left(\omega^{2 a} b_{1}^{2} b_{2}\right)^{2}=\left(\omega q^{-2}\right)^{r(1)}$ for some $r(1) \in \mathbb{Z}_{+}$. Hence $b_{1}=\epsilon_{1} \omega^{-a+r(1)} q^{-\frac{r(1)+r(2)}{2}}$ for some $\epsilon_{1}$ with $\epsilon_{1}^{4}=1$. Hence $\lambda \in \mathcal{B}$, as desired.

$\S 3 . \quad$ Cases of $r(1) \leq 1$

Let $\mathcal{A}_{r(1), r(2)}=\mathcal{A} \cap \mathcal{B}_{r(1), r(2)}$. Then, by Lemma 2.2, we have

$$
\mathcal{A}=\cup_{(r(1), r(2)) \in \mathbb{Z}_{+} \times \mathbb{Z}_{+}} \mathcal{A}_{r(1), r(2)} .
$$


Lemma 3.1. Assume that $r(1) \leq 1$ and $(r(1), r(2)) \neq(0,0)$. Then $\mathcal{A}_{r(1), r(2)}=\emptyset$.

Proof. Let $\lambda=\left(a, \epsilon_{1} \omega^{-a+r(1)} q^{-\frac{r(1)+r(2)}{2}}, \epsilon_{2} q^{r(2)}\right) \in \mathcal{B}_{r(1), r(2)}($ see $(2.11))$. We show $\lambda \notin \mathcal{A}_{r(1), r(2)}$.

Let $v=v_{\lambda}$ (see (2.9)-(2.10)) and assume $\lambda \in \mathcal{A}_{r(1), r(2)}$, i.e., $\operatorname{dim} U v<\infty$ (recall $U v=U^{-} v=\mathcal{L}(\lambda)$ ).

We show $F_{1}^{2} v=0$. Assume $F_{1}^{2} v \neq 0$. By (2.1)-(2.2), we have $E_{112} F_{1}^{2} v=0$. By (2.13) for $n=2$ and the same argument as in Proof of Lemma 2.2, we have $\left(\omega q^{-2}\right)^{r(1)-2}=\left(\omega q^{-2}\right)^{l}$ for some $l \in \mathbb{Z}_{+}$(use the same formula as (2.17) with $F_{1}^{2} v$ in place of $v$ ). This is absurd since $r(1) \leq 1$. Hence $F_{1}^{2} v=0$.

We show $F_{1} v=0$. Assume $F_{1} v \neq 0$. Since $F_{1}^{2} v=0$, by (2.12), similarly to $(2.16)$, we have

$$
0=E_{1} F_{1}^{2} v=-\left(1+\omega^{-1}\right)\left(\epsilon_{1} \omega^{r(1)} q^{-\frac{r(1)+r(2)}{2}}\right)^{-1}\left(\epsilon_{1}^{2} \omega^{2 r(1)} q^{-(r(1)+r(2))}-\omega\right) F_{1} v .
$$

Hence $\epsilon_{1}^{2} \omega^{2 r(1)} q^{-(r(1)+r(2))}=\omega$. This is absurd since $r(1)+r(2)>0$. Hence $F_{1} v=0$.

By $F_{1} v=0$ and (2.12), we have

$$
0=\left(-L_{1}\right) E_{1} F_{1} v=\left(L_{1}^{2}-1\right) v=\left(\epsilon_{1}^{2} \omega^{2 r(1)} q^{-(r(1)+r(2))}-1\right) v
$$

which contradicts $r(1)+r(2)>0$. Hence $\operatorname{dim} U v=\infty$. Hence $\lambda \notin \mathcal{A}_{r(1), r(2)}$, contradiction. Then we have $\mathcal{A}_{r(1), r(2)}=\emptyset$, as desired.

Let $\mathcal{A}_{0,0}^{\prime}=\left\{\left(a, \epsilon_{1}^{\prime} \omega^{-a}, \epsilon_{2}\right) \mid a \in\{0,1,2\}, \epsilon_{1}^{\prime}, \epsilon_{2} \in\{1,-1\}\right\}$. We have the following.

Lemma 3.2. We have $\mathcal{A}_{0,0}=\mathcal{A}_{0,0}^{\prime}$. Moreover, $\operatorname{dim} \mathcal{L}(\lambda)=1$ if and only if $\lambda \in \mathcal{A}_{0,0}^{\prime}$ (see (2.10) for $\left.\mathcal{L}(\lambda)\right)$.

Proof. Use an argument similar to that in Proof of Lemma 3.1 and the fact that $\epsilon_{1}^{2} \in\{ \pm 1\}$ and $\epsilon_{1}^{2} \neq \omega$ (notice that if $r(1)=r(2)=0, \epsilon_{1}^{2}=$ $\left.\epsilon_{1}^{2} \omega^{2 r(1)} q^{-(r(1)+r(2))}\right)$. We also use the fact that for $\lambda \in \mathcal{A}_{r(1), r(1)}$, letting $v=$ $v_{\lambda}(\in \mathcal{L}(\lambda))$ (see $\left.(2.9)-(2.10)\right)$, we have $F_{2}^{r(2)+1} v=0, F_{2}^{i} v \neq 0(0 \leq i \leq r(2))$, $F_{112}^{r(1)+1} v=0$ and $F_{112}^{j} v \neq 0(0 \leq j \leq r(1))$; these equations follow from $(2.16)-(2.17)$.

\section{§4. Main Theorem}

Lemma 4.1. Assume $r(1) \geq 2$. Then $\mathcal{A}_{r(1), r(2)}=\mathcal{B}_{r(1), r(2)}$. 
Proof. It is clear that $\mathcal{A}_{r(1), r(2)} \subset \mathcal{B}_{r(1), r(2)}$. We show $\mathcal{B}_{r(1), r(2)} \subset$ $\mathcal{A}_{r(1), r(2)}$. Let $\lambda=\left(a, \epsilon_{1} \omega^{-a+r(1)} q^{-\frac{r(1)+r(2)}{2}}, \epsilon_{2} q^{r(2)}\right) \in \mathcal{B}_{r(1), r(2)}$ (see (2.11)). Let $v=v_{\lambda}$ (see $(2.9)-(2.10)$ and notice $\left.U v=U^{-} v=\mathcal{L}(\lambda)\right)$. We show $\lambda \in \mathcal{A}_{r(1), r(2)}$, i.e., we show $\operatorname{dim} \mathcal{L}(\lambda)<\infty$. We often use the fact that for $Y \in \Omega\left(U_{m, n}^{+}\right)$with $(m, n) \neq(0,0)$,

$$
E_{1} Y v=E_{2} Y v=0 \quad \Longrightarrow \quad Y v=0 .
$$

A proof of (4.1) is as follows. Since $U^{-} Y v \subset \oplus_{(m, n) \neq(0,0)} \Omega\left(U_{m, n}^{+}\right) v, U Y v=$ $U^{-} Y v$ is a proper submodule of $\mathcal{L}(\lambda)$. By the irreducibility of $\mathcal{L}(\lambda)$, we see $U^{-} Y v=\{0\}$, as desired.

(Strategy of Proof. As mentioned in Remark 1 (see also Remark 5), it suffices to show $\operatorname{dim} V_{i} v<\infty$ for $i=1,2$ (see also Step 5 below). The key of the proof is to show $F_{112}^{r(1)+1} v=0$ (see (4.19)). To prove this equality, we first show $F_{1}^{2} F_{112}^{r(1)-1} v=0$ (see $(4.2)$ ), secondly $F_{1} F_{112}^{r(1)} v=0$ (see (4.6)) and finally $F_{12} F_{112}^{r(1)} v=0$ (see (4.10)). Our main tool is the fact (4.1).)

We proceed in steps. We always use the equations (1.2)-(1.16) and (2.1)(2.7). We also use the equality $E_{112}=E_{1}^{2} E_{2}+\omega q E_{1} E_{2} E_{1}+\omega^{2} q^{2} E_{2} E_{1}^{2}$ (resp. $E_{12}=E_{1} E_{2}-q E_{2} E_{1}$ ) (see (1.9)) for (4.5), (4.9) and (4.18) (resp. (4.4) and (4.8)) below.

Step 1. We show

$$
F_{1}^{2} F_{112}^{r(1)-1} v=0 .
$$

Since $F_{1}^{3}=0$ (see (1.7)), by (1.5), (2.4) and (1.13), we have

$$
E_{2} F_{1}^{2} F_{112}^{r(1)-1} v=0 .
$$

Similarly to (4.3), by (2.6) and (2.1), we also have $E_{12} F_{1}^{2} F_{112}^{r(1)-1} v=0$. Hence, by (4.3), we have

$$
E_{2} E_{1} F_{1}^{2} F_{112}^{r(1)-1} v=0
$$

We see $E_{112} F_{1}^{2} v=0$ by (2.1) and (2.2). Hence, by (2.13) for $n=2$ and the same formula as $(2.17)$ with $F_{1}^{2} v$ in place of $v$, we have $E_{112} F_{112}^{r(1)-1} F_{1}^{2} v=0$. Hence, by (1.13), $E_{112} F_{1}^{2} F_{112}^{r(1)-1} v=0$. Hence, by (4.3)-(4.4), we have

$$
E_{2} E_{1}^{2} F_{1}^{2} F_{112}^{r(1)-1} v=0 .
$$

Recall $E_{1}^{3}=0$ (see (1.6)). Then $E_{1}^{3} F_{1}^{2} F_{112}^{r(1)-1} v=0$. Hence, by (4.5) and (4.1), we have $E_{1}^{2} F_{1}^{2} F_{112}^{r(1)-1} v=0$. Hence, by (4.4) and (4.1), we have $E_{1} F_{1}^{2} F_{112}^{r(1)-1} v=0$. Hence, by (4.3) and (4.1), we have (4.2). 
Step 2. We show

$$
F_{1} F_{112}^{r(1)} v=0
$$

Similarly to (4.3), we have

$$
E_{2} F_{1} F_{112}^{r(1)} v=0 .
$$

By (4.2), (2.1) and (2.6), since $F_{1}^{3}=0$ (see (1.7)), similarly to (4.7), we have $E_{12} F_{1} F_{112}^{r(1)} v=0$. Hence, by (4.7), we have

$$
E_{2} E_{1} F_{1} F_{112}^{r(1)} v=0 .
$$

By (2.2), $E_{112} F_{1} v=0$. Hence, by (2.13) for $n=1$ and the same formula as (2.17) with $F_{1} v$ in place of $v$, we have $E_{112} F_{112}^{r(1)} F_{1} v=0$. Hence, by (1.13), $E_{112} F_{1} F_{112}^{r(1)} v=0$. Hence, by (4.7)-(4.8), we have

$$
E_{2} E_{1}^{2} F_{1} F_{112}^{r(1)} v=0 .
$$

By $(4.7)-(4.9)$ and the same argument as that in the last paragraph of Step 1, we have (4.6).

Step 3. We show

$$
F_{12} F_{112}^{r(1)} v=0 .
$$

By (4.2), (4.6) and (2.3), similarly to (4.3), we have

$$
E_{2} F_{12} F_{112}^{r(1)} v=0 .
$$

Here, for later use, we give some formulas. By (2.2) and (1.15), we have

$$
\left[E_{1}, F_{112}^{m}\right]=-\omega^{2}\left(\omega^{2} q^{-1}\right)^{m} \frac{1-\left(\omega^{2} q^{2}\right)^{m}}{1-\omega^{2} q^{2}} F_{12} F_{112}^{m-1} L_{1}^{-1} .
$$

By (2.1) and (1.12), we have

$$
\left[E_{1}, F_{12}^{2}\right]=\omega^{2} q^{-2} F_{2} F_{12} L_{1}^{-1} .
$$

By (2.3), we have

$$
\left[E_{2}, F_{12}^{2}\right]=-\left(q^{-1}\left(q^{2}-\omega\right) F_{112}+\omega F_{12} F_{1}\right) L_{2} .
$$

Let $x=\epsilon_{1} \omega^{r(1)} q^{-\frac{r(1)+r(2)}{2}}$. By (2.12), we have $L_{1} v=x v$. By (2.1) and (4.12), we have

$$
\begin{aligned}
& E_{1} F_{12} F_{112}^{r(1)} v \\
& =-q^{-1}\left(\omega^{2} q^{-1}\right)^{r(1)} x^{-1} F_{2} F_{112}^{r(1)} v \\
& \quad-\omega^{2}\left(\omega^{2} q^{-1}\right)^{r(1)} \frac{1-\left(\omega^{2} q^{2}\right)^{r(1)}}{1-\omega^{2} q^{2}} x^{-1} F_{12}^{2} F_{112}^{r(1)-1} v .
\end{aligned}
$$


By (4.2), (4.14)-(4.15), (1.13), (2.3)-(2.4) and $F_{1}^{3}=0$ (see (1.7)), we have

$$
E_{2}^{2} E_{1} F_{12} F_{112}^{r(1)} v=0 .
$$

By $F_{12}^{3}=0$ (see (1.16)), (4.12), (4.13) and (4.15), similarly to (4.15), we have

$$
\begin{aligned}
E_{1}^{2} & F_{12} F_{112}^{r(1)} v \\
= & -q^{-1}\left(\omega^{2} q^{-1}\right)^{r(1)} x^{-1} \\
& \cdot\left(-\omega^{2}\left(\omega^{2} q^{-1}\right)^{r(1)} \frac{1-\left(\omega^{2} q^{2}\right)^{r(1)}}{1-\omega^{2} q^{2}}\right) x^{-1} F_{2} F_{12} F_{112}^{r(1)-1} v \\
& -\omega^{2}\left(\omega^{2} q^{-1}\right)^{r(1)} \frac{1-\left(\omega^{2} q^{2}\right)^{r(1)}}{1-\omega^{2} q^{2}} x^{-1} \\
& \cdot \omega^{2} q^{-2}\left(\omega^{2} q^{-1}\right)^{r(1)-1} x^{-1} F_{2} F_{12} F_{112}^{r(1)-1} v \\
= & 0 .
\end{aligned}
$$

Since $E_{112} F_{12} v=0$ (by (2.6)), by (2.14) for $n=1$ and the same formula as (2.17) with $F_{12} v$ in place of $v$, we have $E_{112} F_{112}^{r(1)} F_{12} v=0$. Hence $E_{112} F_{12} F_{112}^{r(1)} v$ $=0$ by (1.15). Hence, by (4.11) and (4.17), we have

$$
E_{1} E_{2} E_{1} F_{12} F_{112}^{r(1)} v=0 .
$$

By (4.11), (4.16)-(4.18) and the same argument as that in the last paragraph of Step 1, we have (4.10).

Step 4. By (4.6) and (4.10), we have

$$
F_{112}^{r(1)+1} v=0 .
$$

Step 5. By the same formula as (2.16), we have $E_{2} F_{2}^{r(2)+1} v=0$. Hence, since $E_{1} F_{2}^{r(2)+1} v=0$, by (4.1), we have

$$
F_{2}^{r(2)+1} v=0 .
$$

By Lemma 1.2 and (4.20), using $\Omega$, we see that $U^{-} v$ is spanned by the elements

$$
F_{1}^{n(1)} F_{112}^{n(112)} F_{12}^{n(12)} F_{2}^{n(2)} v
$$

with $0 \leq n(1) \leq 2, n(112) \in \mathbb{Z}_{+}, 0 \leq n(12) \leq 2$ and $0 \leq n(2) \leq r(2)$. Moreover, by (4.19) and (1.12)-(1.16), we see that for given $n(1), n(12)$ and $n(2)$, there exists $n(112)$ such that the element (4.21) is zero. This implies $\operatorname{dim} \mathcal{L}(\lambda)=\operatorname{dim} U^{-} v<\infty$. Hence $\lambda \in \mathcal{A}_{r(1), r(2)}$. Hence $\mathcal{B}_{r(1), r(2)} \subset \mathcal{A}_{r(1), r(2)}$, which completes the proof.

Now we state our main theorem. 
Theorem 4.1. We have

$$
\mathcal{A}=\mathcal{A}_{0,0}^{\prime} \cup \cup_{r(1)=2}^{\infty} \cup_{r(2)=0}^{\infty} \mathcal{B}_{r(1), r(2)} .
$$

(See also Lemma 2.1.)

Proof. This follows from (3.1) and Lemmas 3.1, 3.2 and 4.1 immediately. (See also Remark 1.)

\section{$\S 5 . \quad$ Additional Remarks}

Here we give some additional remarks.

Remark 7. Difference between $U$ and the (standard) quantum (super) algebras. We show that as a Hopf algebra, $U$ is not isomorphic to any of the quantum algebras $U_{q} \mathfrak{g}$ of the finite dimensional simple Lie algebras $\mathfrak{g}$. We also show that as a Hopf algebra, $U$ is not isomorphic to any of the Hopf algebras $\left(U_{q} \mathfrak{a}\right)^{\sigma}$ associated with the quantum superalgebras $U_{q} \mathfrak{a}$ of the finite dimensional simple Lie superalgebras $\mathfrak{a}$ of type A-G. Here $\left(U_{q} \mathfrak{a}\right)^{\sigma}$ are the Hopf algebras associated with the Hopf superalgebras $U_{q} \mathfrak{a}$ (see $[13,10.1]$ and $[18$, 1.9]). For a Hopf algebra $\mathcal{H}=\left(\mathcal{H}, \Delta_{\mathcal{H}}\right)$, we say that an element $X$ of $\mathcal{H}$ is pre-primitive if $\Delta_{\mathcal{H}}(X) \neq X \otimes X$ and $\Delta_{\mathcal{H}}(X)=X \otimes Y+Z \otimes X$ for some $Y$, $Z \in \mathcal{H}$.

Let $X$ be a pre-primitive element of $U$. We show that

$$
X=y W K_{1}^{c(1)} K_{2}^{c(2)} \sigma^{c(3)}
$$

for some $y \in \mathbb{K}^{\times}, W \in\left\{E_{1}, E_{2}, F_{1}, F_{2}\right\}, c(1), c(2) \in \mathbb{Z}$ and $c(3) \in\{0,1,2\}$. Let $B_{\text {mon }}^{+}$be a basis of $U^{+}$formed by some monomial elements $E_{i(1)} \cdots E_{i(t)}$ with $i(s) \in\{1,2\}(1 \leq s \leq t)$. Let $B^{0}=\left\{K_{1}^{c(1)} K_{2}^{c(2)} \sigma^{c(3)} \mid c(1), c(2) \in \mathbb{Z}, c(3) \in\right.$ $\{0,1,2\}\}$. Using the basis $\left\{\Omega\left(Y^{\prime}\right) J Y^{\prime \prime} \mid Y^{\prime}, Y^{\prime \prime} \in B_{\text {mon }}^{+}, J \in B^{0}\right\}$ of $U$ (see Lemma 1.1 (1)-(2)), we conclude that

$$
\Delta(X)=X \otimes J^{\prime}+J^{\prime \prime} \otimes X
$$

for some $J^{\prime}, J^{\prime \prime} \in B^{0}$. Let $\pi_{-}: U \rightarrow U /\left(U^{0}+U E_{1}+U E_{2}\right)$ and $\pi_{+}: U \rightarrow$ $U /\left(U^{0}+F_{1} U+F_{2} U\right)$ be the canonical maps. We see that $X \in \operatorname{Span}_{\mathbb{K}}\left(U^{0} U^{+}\right) \cup$ $\operatorname{Span}_{\mathbb{K}}\left(U^{-} U^{0}\right)$ since if this is not true, then $\left(\pi_{-} \otimes \pi_{+}\right)(\Delta(X)) \neq 0$, which contradicts (5.2). Notice that if $Y \in \operatorname{Span}_{\mathbb{K}}\left(U^{0} U^{+}\right)=\operatorname{Span}_{\mathbb{K}}\left(U^{+} U^{0}\right)=$ $\Omega^{-1}\left(\operatorname{Span}_{\mathbb{K}}\left(U^{-} U^{0}\right)\right)$ and $\Delta(Y)=\sum_{i} Y_{i}^{(1)} \otimes Y_{i}^{(2)}$, then $\Delta(\Omega(Y))=\sum_{i} \Omega\left(Y_{i}^{(2)}\right)$ $\otimes \Omega\left(Y_{i}^{(1)}\right)$. So we may assume $X \in \operatorname{Span}_{\mathbb{K}}\left(U^{0} U^{+}\right)$. More precisely, we see that 
$X \in U_{m, n}^{+} J$ for some $J \in B^{0}$ and $(m, n) \neq(0,0)$. Moreover, we may also assume $X \in U_{m, n}^{+}$. Using the basis (1.21) of $U^{+}$, we express $X$ as

$$
X=\sum_{(m)} a_{(m)} E_{1}^{m(1)} E_{112}^{m(112)} E_{12}^{m(12)} E_{2}^{m(2)},
$$

where $(m)=(m(1), m(112), m(12), m(2))$ and $a_{(m)} \in \mathbb{K}$. For $(m)$ with $m(1) \geq$ 1 and $m(1)+m(112)+m(12)+m(2) \geq 2$, we have

$$
\begin{aligned}
0 & =\left(E_{1} \otimes E_{1}^{m(1)-1} E_{112}^{m(112)} E_{12}^{m(12)} E_{2}^{m(2)}, \Delta(X)\right) \quad(\text { by }(5.2) \text { and Lemma 1.1) } \\
& =\left(E_{1}^{m(1)} E_{112}^{m(112)} E_{12}^{m(12)} E_{2}^{m(2)}, X\right) \quad(\text { by }(1.8)) \\
& \in \mathbb{K}^{\times} a_{(m)} \quad(\text { by }(1.22) \text { and }(1.19)-(1.20)),
\end{aligned}
$$

whence $a_{(m)}=0$. By the repetition of this argument and (1.17)-(1.18), we have the desired result (5.1).

Let $\widehat{U}$ be $U_{q} \mathfrak{g}$ or $\left(U_{q} \mathfrak{a}\right)^{\sigma}$. By the same argument as above and the use of [4, Proposition 8.28] (see also [6, Lemma 7] and [9, Proposition 3.2]) and [18, Lemmas 10.2.1 and 10.3.1], we also have a result for $\widehat{U}$ similar to the above one for $U$. Then, comparing the pre-primitive elements of $U$ and those of $\widehat{U}$ and using the fact that $E_{1}^{2} \neq 0$ and $E_{1}^{3}=0$ in $U$ (see (1.6) and Lemma 1.2), we see that $U$ is not isomorphic to $\widehat{U}$ as a Hopf algebra, as desired.

Remark $8 . \quad$ Finite dimensional $\mathbb{Z} / 3 \mathbb{Z}$-quantum group. Here we give a finite dimensional version $\mathbf{u}$ of $U$, which is a counterpart of the finite dimensional quantum groups at roots of 1 introduced by Lusztig [11].

We first give some facts on $U$. Let $P: U \otimes U \rightarrow U \otimes U$ be the $\mathbb{K}$ algebra homomorphism defined by $P(X \otimes Y)=Y \otimes X(X, Y \in U)$. Then we have $\Delta \circ \Omega=(\Omega \otimes \Omega) \circ P \circ \Delta$. (Using this, in a way similar to that for $[4$, Proposition 6.12], we can prove Lemma 1.1 (4).) We also have $\Omega \circ S=S^{-1} \circ \Omega$ and $\varepsilon \circ \Omega=\varepsilon$. (See also $[4,3.8]$.) For all $X, Y \in \operatorname{Span}_{\mathbb{K}}\left(U^{+} U^{0}\right)$, we have

$$
(X, 1)=\varepsilon(X) \text { and }(S(X), Y)=(X, S(X)) .
$$

(see also [4, Exercise 6.16]). For all $X_{1}, X_{2} \in \operatorname{Span}_{\mathbb{K}}\left(U^{+} U^{0}\right)$, writing $((\Delta \otimes$ id) $\circ \Delta)\left(X_{i}\right)=\sum_{j} X_{i, j} \otimes X_{i, j}^{\prime} \otimes X_{i, j}^{\prime \prime}(i \in\{1,2\})$, we have

$$
\Omega\left(X_{2}\right) X_{1}=\sum_{s, t}\left(S^{-1}\left(X_{1, s}\right), X_{2, t}^{\prime \prime}\right)\left(X_{1, s}^{\prime \prime}, X_{2, t}\right) X_{1, s}^{\prime} \Omega\left(X_{2, t}^{\prime}\right)
$$

(see also [1, Section 13], [4, $6.17(3)]$ and [18, 1.4 and 2.4]).

Set $\mathbb{A}:=\mathbb{C}\left[q, q^{-1},\left(q^{2}-1\right)^{-1},\left(q^{2}-\omega\right)^{-1}\right](\subset \mathbb{K})$. Let $U_{\mathbb{A}}$ be the $\mathbb{A}$-submodule of $U$ having the $\mathbb{A}$-basis formed by the elements (2.8). By (1.2)-(1.5), (1.9)- 
(1.18) and (2.1)-(2.7), we can see that $U_{\mathbb{A}} U_{\mathbb{A}} \subset U_{\mathbb{A}}, \Delta\left(U_{\mathbb{A}}\right) \subset U_{\mathbb{A}} \otimes_{\mathbb{A}} U_{\mathbb{A}}$ $(\subset U \otimes U), S\left(U_{\mathbb{A}}\right)=U_{\mathbb{A}}, \varepsilon\left(U_{\mathbb{A}}\right)=\mathbb{A}$ and $\Omega\left(U_{\mathbb{A}}\right)=U_{\mathbb{A}}$. (For the last equality, we should assume $\left(q^{2}-1\right)^{-1}\left(q^{2}-\omega\right)^{-1} \in \mathbb{A}$.) Then $U_{\mathbb{A}}$ has an $\mathbb{A}$-Hopf algebra structure $\left(U_{\mathbb{A}}, \Delta, S, \varepsilon\right)$.

Let $\zeta \in \mathbb{C}$ be such that $\zeta\left(\zeta^{2}-1\right)\left(\zeta^{2}-\omega\right) \neq 0$. Assume that there exists $e^{\prime} \in \mathbb{N}$ such that $\zeta^{e^{\prime}}=1$. Set $e:=\min \left\{e^{\prime} \in \mathbb{N} \mid \zeta^{e^{\prime}}=1\right\}$. Then $\zeta$ is a primitive $e$-th root of unity. Regard $\mathbb{C}$ as an $\mathbb{A}$-algebra via the $\mathbb{C}$-algebra homomorphism $\mathbb{A} \rightarrow \mathbb{C}$ that takes $q$ to $\zeta$. Set $\mathbf{U}:=U_{\mathbb{A}} \otimes_{\mathbb{A}} \mathbb{C}$. The argument in the previous paragraph implies that $\mathbf{U}$ has a $\mathbb{C}$-Hopf algebra structure $(\mathbf{U}, \Delta, S, \varepsilon)$, where we denote again by $\Delta, S$ and $\varepsilon$ the maps induced from the above ones. We also denote by $\Omega$ the $\mathbb{C}$-algebra automorphism of $\mathbf{U}$ induced from the above one. Let $\mathbf{U}^{+}$be the subalgebra $\left(U_{\mathbb{A}} \cap U^{+}\right) \otimes_{\mathbb{A}} \mathbb{C}$ of $\mathbf{U}$. Let $\mathbf{U}^{\geq 0}$ be the Hopf subalgebra $\left(U_{\mathbb{A}} \cap \operatorname{Span}_{\mathbb{K}}\left(U^{+} U^{0}\right)\right) \otimes_{\mathbb{A}} \mathbb{C}$ of $\mathbf{U}$. Notice that $\mathbf{U}$ also has a property similar to Lemma 1.1 (1).

Denote again by $($,$) the bilinear form on \mathbf{U}^{\geq 0}$ induced from the one on $\operatorname{Span}_{\mathbb{K}}\left(U^{+} U^{0}\right)$ (this is well-defined; see (1.22), (1.19)-(1.20) and Lemma $1.1(4))$. Let $\mathbf{I}^{\geq 0}=\operatorname{ker}($,$) . By (1.8) and (5.3), we see that$

$$
\left\{\begin{array}{l}
\mathbf{U}^{\geq 0} \mathbf{I}^{\geq 0} \mathbf{U}^{\geq 0}=\mathbf{I}^{\geq 0}, \Delta\left(\mathbf{I}^{\geq 0}\right) \subset \mathbf{I}^{\geq 0} \otimes \mathbf{U}^{\geq 0}+\mathbf{U}^{\geq 0} \otimes \mathbf{I}^{\geq 0} \\
S\left(\mathbf{I}^{\geq 0}\right)=\mathbf{I}^{\geq 0}, \quad \varepsilon\left(\mathbf{I}^{\geq 0}\right)=\{0\} .
\end{array}\right.
$$

Let $\mathbf{J}_{1}$ (resp. $\mathbf{J}_{2}$ ) be the subspace of $\mathbf{U}$ spanned by $\mathbf{I}^{\geq 0} \Omega\left(\mathbf{U}^{+}\right)\left(\right.$resp. $\mathbf{U}^{+} \Omega\left(\mathbf{I}^{\geq 0}\right)$ ). By (5.4) and formulas in the second paragraph of this remark, we also have the same formulas as in (5.4) with $\mathbf{U}$ and $\mathbf{J}_{i}$ in place of $\mathbf{U}^{\geq 0}$ and $\mathbf{I}^{\geq 0}(i \in\{1,2\})$. Set $\mathbf{u}=\mathbf{u}_{\zeta}:=\mathbf{U} /\left(\mathbf{J}_{1}+\mathbf{J}_{2}\right)$. Then $\mathbf{u}$ can be regarded as a $\mathbb{C}$-Hopf algebra. (This construction is similar to [12, 3.1.1 (a)-(e)] and [18, Theorem 2.9.4].)

Set $x:=\min \left\{x^{\prime} \in \mathbb{N} \mid\left(\omega \zeta^{-2}\right)^{x^{\prime}}=1\right\}$ and $y:=\min \left\{y^{\prime} \in \mathbb{N} \mid \zeta^{2 y^{\prime}}=1\right\}$. If $X$ is of (2.8), we denote $X \otimes_{\mathbb{A}} \mathbb{C}+\left(\mathbf{J}_{1}+\mathbf{J}_{2}\right) \in \mathbf{u}$ again by $X$. Using formulas between (1.2) and (2.8), especially (1.22) and Lemma 1.1 (4), we see that the elements denoted as (2.8) with $0 \leq m(1), m(12), c(3), n(1), n(12) \leq 2$, $0 \leq m(112), n(112) \leq x-1,0 \leq m(2), n(2) \leq y-1$ and $0 \leq c(1), c(2) \leq e-1$ form a $\mathbb{C}$-basis of $\mathbf{u}$. Then we have $\operatorname{dim} \mathbf{u}=3^{5} e^{2} x^{2} y^{2}$.

Similarly we have the results below.

Let $\mathbf{u}^{\geq 0}$ be the Hopf subalgebra $\mathbf{U}^{\geq 0}+\left(\mathbf{J}_{1}+\mathbf{J}_{2}\right)$ of $\mathbf{u}$. Identify the dual Hopf algebra $\left(\mathbf{u}^{\geq 0}\right)^{*}$ of $\mathbf{u}^{\geq 0}$ with $\mathbf{u}^{\geq 0}$ itself via the non-degenerate bilinear form on $\mathbf{u}^{\geq 0}$ induced from (, ). Then, using the Drinfeld [1, Section 13] quantum double $D\left(\mathbf{u}^{\geq 0}\right)=\mathbf{u}^{\geq 0} \otimes\left(\mathbf{u}^{\geq 0}\right)^{*}$ (see also [15] and [18, Proposition 1.7.1, Theorem 2.9.4 and Lemma 2.9.10]), by (1.22) and Lemma 1.1 (4), we can see that $\mathbf{u}$ is a quasi- 
triangular Hopf algebra whose universal $R$-matrix $\mathcal{R} \in \mathbf{u} \otimes \mathbf{u}$ is given by

$$
\begin{aligned}
\mathcal{R}= & \left(\sum_{m(1)=0}^{2} \frac{\left(E_{1} \otimes F_{1}\right)^{m(1)}}{\Phi(m(1), \omega)}\right) \cdot\left(\sum_{m(112)=0}^{x-1} \frac{\left(E_{112} \otimes F_{112}\right)^{m(112)}}{\Phi\left(m(112), \omega \zeta^{-2}\right)}\right) \\
& \cdot\left(\sum_{m(12)=0}^{2} \frac{\left(E_{12} \otimes F_{12}\right)^{m(12)}}{\Phi(m(12), \omega)}\right) \cdot\left(\sum_{m(2)=0}^{y-1} \frac{\left(E_{2} \otimes F_{2}\right)^{m(2)}}{\Phi\left(m(2), \zeta^{2}\right)}\right) \\
& \cdot\left(\sum_{i(1), j(1)=0}^{e-1} \frac{\zeta^{i(1) j(1)} K_{1}^{i(1)} \otimes\left(K_{1}^{-2} K_{2}^{-1}\right)^{j(1)}}{e}\right) \\
& \cdot\left(\sum_{i(2), j(2)=0}^{e-1} \frac{\zeta^{i(2) j(2)} K_{2}^{i(2)} \otimes\left(K_{1}^{-1}\right)^{j(2)}}{e}\right) \cdot\left(\sum_{s, t=0}^{2} \frac{\omega^{s t} \sigma^{s} \otimes \sigma^{t}}{3}\right) .
\end{aligned}
$$

As a $\mathbb{C}$-algebra, $\mathbf{u}$ can also be defined with the generators denoted as (1.1) and the same relations as $(1.2)-(1.7)$ with $\zeta$ in place of $q$ and the relations:

$$
K_{1}^{e}=K_{2}^{e}=1, E_{112}^{x}=F_{112}^{x}=0 \text { and } E_{2}^{y}=F_{2}^{y}=0
$$

and

$$
\left\{\begin{array}{l}
E_{112} E_{12}=\omega \zeta E_{112} E_{12}, F_{112} F_{12}=\omega \zeta F_{112} F_{12} \text { if } \zeta \in\{\sqrt{-1},-\sqrt{-1}\} \\
E_{12}^{3}=F_{12}^{3}=0 \text { if } \zeta \in\{\omega, \sqrt{-1},-\sqrt{-1},-\omega\}
\end{array}\right.
$$

As for (5.5), see Remark 4. We can show that (5.5) cannot be dropped.

Remark 9. We can consider a Drinfeld [1]-type $h$-adic topological version $U_{h}$ of $U$ (by replacing $q^{\frac{1}{2}}, K_{i}$ and $F_{i}$ with $e^{\frac{h}{2}}, e^{h H_{i}}$ and $\left(q^{-1}-q\right)^{-1} F_{i}$ respectively). We can also give an explicit formula of a universal $R$-matrix of $U_{h}$. However $U_{h}$ is a topological not $\mathbb{C}[[h]]$ - but $\mathbb{C}((h))$-algebra, where $\mathbb{C}[[h]]$ is the $\mathbb{C}$-algebra of the formal power series in $h$ and $\mathbb{C}((h))$ is the quotient field of $\mathbb{C}[[h]]$. In particular, it seems to be impossible to take a 'good' limitation $\lim _{h \rightarrow 0} U_{h}\left(\right.$ or $\left.\lim _{q \rightarrow 1} U\right)$.

Remark 10. Finite dimensional irreducible representations of $U_{q} \mathrm{osp}(3 \mid 2)$. As mentioned in Introduction, our method for $U$ can also be applied to classify the finite dimensional irreducible representations of $U_{q} \operatorname{osp}(3 \mid 2)$. Here we explain about it.

Let

$$
\bar{A}=\left(\begin{array}{ll}
\bar{a}_{11} & \bar{a}_{12} \\
\bar{a}_{21} & \bar{a}_{22}
\end{array}\right)=\left(\begin{array}{cc}
0 & 1 \\
-2 & 2
\end{array}\right)
$$


Let $\bar{p}(1)=1$ and $\bar{p}(2)=0$.

The Lie superalgebra $\operatorname{osp}(3 \mid 2)$ is also denoted as $B(1,1)$ and the following is the Dynkin diagram of $\operatorname{osp}(3 \mid 2)=B(1,1)$ (see $[8]$ ).

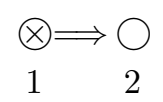

Let

$$
\bar{q}_{1}=q^{-1} \quad \text { and } \quad \bar{q}_{2}=q^{\frac{1}{2}} .
$$

Let $\bar{U}=U_{q} \operatorname{osp}(3 \mid 2)$ be a $\mathbb{K}$-algebra defined with generators $\bar{K}_{i}^{ \pm 1}, \bar{E}_{i}, \bar{F}_{i}$ $(i \in\{1,2\})$ and defining relations $\bar{K}_{i} \bar{K}_{i}^{-1}=\bar{K}_{i}^{-1} \bar{K}_{i}=1, \bar{K}_{i} \bar{K}_{j}=\bar{K}_{j} \bar{K}_{i}$, $\bar{K}_{i} \bar{E}_{j} \bar{K}_{i}^{-1}=\bar{q}_{i}^{\bar{a}_{i j}} \bar{E}_{j}, \bar{K}_{i} \bar{F}_{j} \bar{K}_{i}^{-1}=\bar{q}_{i}^{-\bar{a}_{i j}} \bar{F}_{j}, \bar{E}_{i} \bar{F}_{j}-(-1)^{\bar{p}(i) \bar{p}(j)} \bar{F}_{j} \bar{E}_{i}=\delta_{i j}\left(\bar{q}_{i}-\right.$ $\left.\bar{q}_{i}^{-1}\right)^{-1}\left(\bar{K}_{i}-\bar{K}_{i}^{-1}\right), \bar{E}_{1}^{2}=0, \bar{E}_{2}^{3} \bar{E}_{1}-\left(q+1+q^{-1}\right) \bar{E}_{2}^{2} \bar{E}_{1} \bar{E}_{2}+\left(q+1+q^{-1}\right) \bar{E}_{2} \bar{E}_{1} \bar{E}_{2}^{2}-$ $\bar{E}_{1} \bar{E}_{2}^{3}=0, \bar{F}_{1}^{2}=0, \bar{F}_{2}^{3} \bar{F}_{1}-\left(q+1+q^{-1}\right) \bar{F}_{2}^{2} \bar{F}_{1} \bar{F}_{2}+\left(q+1+q^{-1}\right) \bar{F}_{2} \bar{F}_{1} \bar{F}_{2}^{2}-\bar{F}_{1} \bar{F}_{2}^{3}=0$. Then $\bar{U}$ can be viewed as a Hopf superalgebra in a standard way.

Let $\bar{E}_{12}=\bar{E}_{1} \bar{E}_{2}-q \bar{E}_{2} \bar{E}_{1}, \bar{E}_{122}=\bar{E}_{12} \bar{E}_{2}-\bar{E}_{2} \bar{E}_{12}, \bar{F}_{12}=\bar{F}_{1} \bar{F}_{2}-q \bar{F}_{2} \bar{F}_{1}$ and $\bar{F}_{122}=\bar{F}_{12} \bar{F}_{2}-\bar{F}_{2} \bar{F}_{12}$. Then $\bar{E}_{122}^{2}=\bar{F}_{122}^{2}=0$. By an argument similar to that in the proof of $[18$, Theorem 10.5.1], we see that the elements

$$
\bar{F}_{1}^{\bar{m}(1)} \bar{F}_{12}^{\bar{m}(12)} \bar{F}_{122}^{\bar{m}(122)} \bar{F}_{2}^{\bar{m}(2)} \bar{K}_{1}^{\bar{c}(1)} \bar{K}_{2}^{\bar{c}(2)} \bar{E}_{1}^{\bar{n}^{(1)}} \bar{E}_{12}^{\bar{n}(12)} \bar{E}_{122}^{\bar{n}(122)} \bar{E}_{2}^{\bar{n}(2)}
$$

$\left(\bar{m}(1), \bar{m}(122), \bar{n}(1), \bar{n}(122) \in\{0,1\}, \bar{m}(12), \bar{m}(2), \bar{n}(12), \bar{n}(2) \in \mathbb{Z}_{+}, \bar{c}(1), \bar{c}(2)\right.$ $\in \mathbb{Z})$ form a $\mathbb{K}$-basis of $\bar{U}$.

Let $\bar{U}^{-}$be a subalgebra of $\bar{U}$ generated by $\bar{F}_{1}$ and $\bar{F}_{2}$. By the same argument as that for $\mathcal{L}\left(a, b_{1}, b_{2}\right)$ (see $\S 2$ ), we see that for $\bar{b}_{1}, \bar{b}_{2} \in \mathbb{K}^{\times}$, there exists a unique (non-zero) irreducible left $\bar{U}$-module $\overline{\mathcal{L}}\left(\bar{b}_{1}, \bar{b}_{2}\right)$ such that $\bar{K}_{i} \bar{v}=$ $\bar{b}_{i} \bar{v}, \bar{E}_{i} \bar{v}=0$ and $\overline{\mathcal{L}}\left(\bar{b}_{1}, \bar{b}_{2}\right)=\bar{U}^{-} \bar{v}$ for some $\bar{v} \in \overline{\mathcal{L}}\left(\bar{b}_{1}, \bar{b}_{2}\right)$. Let $\overline{\mathcal{A}}=\left\{\left(\bar{b}_{1}, \bar{b}_{2}\right) \in\right.$ $\left.\left(\mathbb{K}^{\times}\right)^{2} \mid \operatorname{dim} \overline{\mathcal{L}}\left(\bar{b}_{1}, \bar{b}_{2}\right)<\infty\right\}$. Then we have the same result as Lemma 2.1. For $(\bar{r}(1), \bar{r}(2)) \in\left(\mathbb{Z}_{+}\right)^{2}$, let $\overline{\mathcal{B}}_{\bar{r}(1), \bar{r}(2)}=\left\{\left(\bar{b}_{1}, \bar{b}_{2}\right) \in\left(\mathbb{K}^{\times}\right)^{2} \mid \bar{b}_{1}^{2} \bar{b}_{2}^{2}=\left(-q^{-1}\right)^{\bar{r}(1)}, \bar{b}_{2}^{2}=\right.$ $\left.q^{\bar{r}(2)}\right\}$. Let $\overline{\mathcal{A}}_{\bar{r}(1), \bar{r}(2)}=\overline{\mathcal{A}} \cap \overline{\mathcal{B}}_{\bar{r}(1), \bar{r}(2)}$. Since

$$
\bar{E}_{12} \bar{F}_{12}+\bar{F}_{12} \bar{E}_{12}=q \frac{\bar{K}_{1} \bar{K}_{2}-\bar{K}_{1}^{-1} \bar{K}_{2}^{-1}}{\bar{q}_{2}-\bar{q}_{2}^{-1}},
$$

by an argument similar to that in Proof of Lemma 2.2, we have $\overline{\mathcal{A}}=\cup \overline{\mathcal{A}}_{\bar{r}(1), \bar{r}(2)}$.

By an argument similar to that in Proofs of Lemmas 3.1-3.2, we see that $\overline{\mathcal{A}}_{0,0}=\left\{\left(\bar{b}_{1}, \bar{b}_{2}\right) \mid \bar{b}_{1}^{2}=\bar{b}_{2}^{2}=1\right\}=\left\{\left(\bar{b}_{1}, \bar{b}_{2}\right) \mid \operatorname{dim} \overline{\mathcal{L}}\left(\bar{b}_{1}, \bar{b}_{2}\right)=1\right\}$ and that if $\bar{r}(1) \in$ $\{0,1\}$ and $(\bar{r}(1), \bar{r}(2)) \neq(0,0)$, then $\overline{\mathcal{A}}_{\bar{r}(1), \bar{r}(2)}=\emptyset$. It also follows that if $\bar{r}(1) \geq 2$, then $\overline{\mathcal{A}}_{\bar{r}(1), \bar{r}(2)}=\overline{\mathcal{B}}_{\bar{r}(1), \bar{r}(2)}$. This can be proved in an argument similar to that in Proof of Lemma 4.1; more precisely, in such a way that we first show $\bar{F}_{1} \bar{F}_{12}^{\bar{r}(1)-1} \bar{v}=0$, secondly $\bar{F}_{1} \bar{F}_{12}^{\bar{r}(1)} \bar{v}=0$ and finally $\bar{F}_{12}^{\bar{r}(1)+1} \bar{v}=0$. 


\section{Acknowledgements}

The author would like to thank P. Deligne for informative comments and posing a question, which inspired the author to study the subject of this paper. The author also thanks T. Tanisaki and H.-J. Schneider for valuable discussions, M. Takeuchi for comments related to Remark 7 and I. Heckenberger for informing the author about the papers [2] and [3]. The author also thanks Y.M. Zou for useful comments and encouragement to write this paper. The author would like to express his thanks to the referee for the careful reading and the valuable comments.

\section{References}

[1] V. G. Drinfeld, Quantum groups, in Proceedings of the International Congress of Mathematicians, Vol. 1, 2 (Berkeley, Calif., 1986), 798-820, Amer. Math. Soc., Providence, RI.

[2] I. Heckenberger, Finite dimensional rank 2 Nichols algebras of diagonal type I: Examples, Preprint, math.QA/0402350.

[3] - Classifications of arithmetic root systems of rank 3, Preprint, math.QA/0509145.

[4] J. C. Jantzen, Lectures on quantum groups, Amer. Math. Soc., Providence, RI, 1996.

[5] M. Jimbo, A $q$-difference analogue of $U(G)$ and the Yang-Baxter equation, Lett. Math. Phys. 10 (1985), no. 1, 63-69.

[6] A. N. Kirillov and N. Reshetikhin, $q$-Weyl group and a multiplicative formula for universal $R$-matrices, Comm. Math. Phys. 134 (1990), no. 2, 421-431.

[7] S. M. Khoroshkin and V. N. Tolstoy, Universal $R$-matrix for quantized (super)algebras, Comm. Math. Phys. 141 (1991), no. 3, 599-617.

[8] V. G. Kac, Lie superalgebras, Advances in Math. 26 (1977), no. 1, 8-96.

[9] S. Z. Levendorskii and Ya. S. Soibelman, Some applications of the quantum Weyl groups, J. Geom. Phys. 7 (1990), no. 2, 241-254.

[10] J. R. Links and M. D. Gould, Two variable link polynomials from quantum supergroups, Lett. Math. Phys. 26 (1992), no. 3, 187-198.

[11] G. Lusztig, Quantum groups at roots of 1, Geom. Dedicata 35 (1990), no. 1-3, 89-113.

[12] Introduction to quantum groups, Progr. Math., 110, Birkhäuser Boston, Boston, MA, 1993

[13] S. Majid, Foundations of quantum group theory, Cambridge Univ. Press, Cambridge, 1995.

[14] M. Rosso, Finite-dimensional representations of the quantum analog of the enveloping algebra of a complex simple Lie algebra, Comm. Math. Phys. 117 (1988), no. 4, 581-593.

[15] , An analogue of P.B.W. theorem and the universal $R$-matrix for $U_{h} \operatorname{sl}(N+1)$, Comm. Math. Phys. 124 (1989), no. 2, 307-318.

[16] D. De Wit, J. R. Links and L. H. Kauffman, On the Links-Gould invariant of links, J. Knot Theory Ramifications 8 (1999), no. 2, 165-199.

[17] H. Yamane, A Poincaré-Birkhoff-Witt theorem for quantized universal enveloping algebras of type $A_{N}$, Publ. Res. Inst. Math. Sci. 25 (1989), no. 3, 503-520.

[18] _ , Quantized enveloping algebras associated with simple Lie superalgebras and their universal R-matrices, Publ. Res. Inst. Math. Sci. 30 (1994), no. 1, 15-87.

[19] C. N. Yang and M. L. Ge (ed.), Braid group, knot theory and statistical mechanics, World Sci. Publishing, Teaneck, NJ, 1989. 